\title{
CONHECIMENTOS, ATITUDES E PRÁTICA DE HOMENS SOBRE INFECÇÕES SEXUALMENTE TRANSMISSÍVEIS
}

\author{
Carla Muriele Andrade Santos ${ }^{1}$, José Deivid Santos Oliveira ${ }^{1}$, Shirley Verônica Melo Almeida Lima ${ }^{2}$, Allan \\ Dantas dos Santos ${ }^{3}$, Marco Aurélio de Oliveira Góes ${ }^{4}$, Leilane Barbosa de Sousa ${ }^{5}$
}

\begin{abstract}
RESUMO: Objetivos: analisar conhecimentos, atitudes e práticas de homens acerca das infecções sexualmente transmissíveis e o uso do preservativo. Método: estudo descritivo, transversal com abordagem quantitativa com caminhoneiros do município de Itabaiana, Sergipe. A coleta de dados ocorreu entre janeiro e março de 2017 com 75 caminhoneiros. Após a coleta de dados, as entrevistas foram transcritas, analisadas e classificadas pelo conhecimento, atitude e prática dos caminhoneiros. Resultados: dos caminhoneiros, 74 (98,6\%) possuíam conhecimento adequado acerca das infecções, porém foi possível identificar que as atitudes e as práticas não se apresentavam de maneira adequada, principalmente no que diz respeito ao comportamento sexual e os antecedentes de infecções sexualmente transmissíveis. Conclusão: intervenções efetivas, subsidiadas por planejamento estratégico situacional, devem ser estimuladas para a população estudada em diferentes regiões brasileiras. Assim, reduzem cadeias de transmissão das doenças e promovem saúde.
\end{abstract}

DESCRITORES: Infecções sexualmente transmissíveis; Preservativos; Conhecimentos, Atitudes e prática em saúde.

\section{MEN'S KNOWLEDGE, ATTITUDES AND PRACTICE REGARDING SEXUALLY TRANSMITTED DISEASES}

ABSTRACT: Objectives: to analyze men's knowledge, attitudes and practices regarding sexually transmitted infections and condom use. Method: a descriptive, transversal study with a quantitative approach, with truck drivers in the city of Itabaiana, in the Brazilian State of Sergipe. Data collection took place between January and March 2017, with 75 truck drivers. After data collection, the interviews were transcribed, analyzed and classified in terms of the truck drivers' knowledge, attitudes and practice. Results: of the truck drivers, 74 (98.6\%) possessed adequate knowledge regarding the infections; however, it was possible to identify that their attitudes and practices were not adequate - mainly in regard to sexual behavior and previous infection with sexually transmitted infections. Conclusion: effective interventions, supported by situational strategic planning, must be encouraged for the population studied in different regions of Brazil. This would reduce chains of transmission of the diseases and promote health.

DESCRIPTORS: Sexually Transmitted Infections; Condoms; Health Knowledge, Attitudes, Practice.

\section{CONOCIMIENTOS, ACTITUDES Y PRÁCTICA DE HOMBRES ACERCA DE INFECCIONES SEXUALMENTE TRANSMISIBLES}

RESUMEN: Objetivos: analizar conocimientos, actitudes y prácticas de hombres acerca de las infecciones sexualmente transmisibles y el uso del preservativo. Método: estudio descriptivo, transversal de abordaje cuantitativo con camioneros del municipio de Itabaiana, Sergipe. La obtención de datos ocurrió entre enero y marzo de 2017 con 75 camioneros. Después de la obtención de datos, se transcribieron las entrevistas, y fueron analizadas y clasificadas por el conocimiento, actitud y práctica de los camioneros. Resultados: de los camioneros, $74(98,6 \%)$ presentaban conocimiento apropiado acerca de las infecciones, sin embargo fue posible identificar que las actitudes y las prácticas no eran adecuadas, principalmente con relación al comportamiento sexual y a los antecedentes de infecciones sexualmente transmisibles. Conclusión: intervenciones efectivas, subsidiadas por planeamiento estratégico situacional deben ser estimuladas para la población estudiada en diferentes regiones brasileñas. Así, disminuyen las cadenas de transmisión de enfermedades y se promueve salud.

DESCRIPTORES: Infecciones sexualmente transmisibles; Preservativos; Conocimientos, actitudes y prácticas en salud.

${ }^{1}$ Discente de Enfermagem. Universidade Federal de Sergipe. Lagarto, SE, Brasil.

${ }^{2}$ Enfermeira. Mestra em Ciências da Saúde. Docente de Enfermagem da Universidade Federal de Sergipe. Lagarto, SE, Brasil.

${ }^{3}$ Enfermeiro. Doutor em Ciências da Saúde. Docente de Enfermagem da Universidade Federal de Sergipe. Lagarto, SE, Brasil. ${ }^{4}$ Médico. Mestre em Saúde e Ambiente. Docente da Universidade Federal de Sergipe. Lagarto, SE, Brasil.

${ }^{5}$ Enfermeira. Doutora em Enfermagem. Docente de Enfermagem da Universidade da Integração Internacional da Lusofonia Afro-Brasileira. Redenção, CE, Brasil. 


\section{INTRODUÇÃO}

As Infecções Sexualmente Transmissíveis (IST) constituem importante agravo de saúde pública, pois são responsáveis por causar impacto na qualidade de vida das pessoas, nas relações pessoais, familiares e sociais ${ }^{(1-2)}$.

Conforme a Organização Mundial da Saúde (OMS) $)^{(1)}$, mais de um milhão de pessoas adquirem IST diariamente e a cada ano, estima-se que 500 milhões de pessoas adquirem uma das IST curáveis (gonorreia, clamídia, sífilis e tricomoníase).

No mundo, a infecção pelo Papilomavírus Humano (HPV), por exemplo, causa 530.000 casos de câncer de colo uterino e 275.000 mortes por essa doença/ano; a sífilis gestacional, por sua vez, causa aproximadamente 300.000 mortes fetais e neonatais/ano e coloca 215.000 recém-nascidos sob o risco de morte prematura, baixo peso ao nascimento ou sífilis congênita; e calcula-se, ainda, que 530 milhões de pessoas estejam infectadas com o vírus do herpes genital ${ }^{(1)}$.

Segundo os dados divulgados na $21^{\mathrm{a}}$ Conferência Mundial sobre Aids (Síndrome da Imunodeficiência Adquirida), 30 milhões de mortes já foram causadas por esta síndrome; cerca de 36.7 milhões de pessoas vivem com o vírus da imunodeficiência humana (HIV) no mundo e apenas 17 milhões recebem tratamento ${ }^{(3)}$. No Brasil, foram notificados, de 1980 a julho de 2016, 842.710 casos de Aids $^{(4)}$, sinalizando aumento expressivo, ao longo do tempo, na taxa de detecção de Aids entre os homens e redução entre as mulheres ${ }^{(5)}$.

Conforme a OMS, em 2014 a relação sexual desprotegida foi considerada um fator de risco importante à transmissão das IST e do HIV em todo o mundo(6). O preservativo tem sido a forma de profilaxia mais divulgada pelas políticas de saúde, por seu elevado grau de proteção frente ao risco do aparecimento das IST, sobretudo o HIV/Aids, pois ele pode reduzir os ciclos de contaminações ${ }^{(7)}$.

O uso correto e frequente do preservativo está diretamente relacionado à questão de gênero como fator determinante no desenvolvimento da autonomia pessoal, além de ser uma das estratégias de promoção da saúde sexual e reprodutiva, especialmente nas mulheres. O desenvolvimento de habilidades pessoais define o que as mulheres sabem, pensam e praticam acerca de comportamentos sexuais e uso do preservativo ${ }^{(8)}$.

Diante da prevalente problemática das IST no Brasil, os caminhoneiros se caracterizam como um grupo populacional que apresenta vulnerabilidade acrescida frente às IST e HIV, fato que pode influenciar no processo endêmico de disseminação das mesmas ${ }^{(9)}$.

A vulnerabilidade às IST está relacionada à exposição ao risco em virtude, principalmente, da falta de conhecimentos, atitudes e práticas favoráveis à saúde sexual. A vulnerabilidade acrescida em caminhoneiros decorre da grande mobilidade física, além de comportamentos de risco assumidos frente às diversas situações vivenciadas, entre elas a que mais se destaca é a prática do sexo com diversos parceiros ocasionais desprotegida ${ }^{(1,9-10)}$.

Neste contexto de vulnerabilidade, os caminhoneiros merecem atenção específica das ações de saúde, não no sentido de resgatar a antiga noção de grupos de risco, mas sim com o objetivo de observar questões que se tornam imprescindíveis para o planejamento e a implementação de políticas e programas voltados para grupos que vivenciam contextos de maior vulnerabilidade.

Logo, o estudo objetivou analisar conhecimentos, atitudes e práticas de homens acerca das IST e do uso do preservativo.

\section{MÉTODO}

Estudo descritivo, transversal, com abordagem quantitativa realizado no município de Itabaiana, Sergipe. O município foi escolhido por ser a Capital Nacional do Caminhão, por meio da Lei ${ }^{\circ}{ }^{13.044}$, de 19 de novembro de 2014. Segundo o Projeto de Lei 7132/14, Itabaiana tem pouco mais de 100 mil habitantes e cerca de seis mil caminhões emplacados, caracterizando a maior taxa do Brasil ${ }^{(11-12)}$. Neste 
contexto, a cidade possui um número significativo de profissionais caminhoneiros.

A coleta de dados ocorreu entre janeiro e março de 2017 em postos de combustíveis e empresas transportadoras, locais de maior concentração de caminhoneiros. Os critérios de inclusão foram: ser maior de 18 anos e exercer a profissão de caminhoneiro há mais de 6 meses.

A amostra do estudo foi por conveniência, composta por 75 caminhoneiros. Inicialmente foram realizados 10 estudos pilotos. Após estes, o instrumento de coleta de dados foi adaptado. Posteriormente, foram entrevistados os 75 caminhoneiros. O formulário foi composto por 34 questões, que analisavam aspectos sociodemográficos e de trabalho; conhecimento acerca das IST, HIV/Aids; conhecimento sobre transmissão e prevenção das IST; fontes de conhecimento; testagem de HIV, práticas sexuais, comportamento sexual e uso de preservativo pelos caminhoneiros. O instrumento utilizado foi uma adaptação do questionário principal da Pesquisa de Conhecimentos, Atitudes e Práticas na População Brasileira $^{(6)}$.

Após a coleta de dados, os dados foram tabulados no programa Excel 2013 e analisados descritivamente de acordo com as seguintes temáticas: conhecimentos, atitudes e práticas de homens acerca das IST e do uso do preservativo.

A pesquisa obedeceu à resolução $\mathrm{n}^{\circ} 466$, de 12 de dezembro de $2012^{(13)}$ e foi aprovada pelo Comitê de Ética em Pesquisa da Universidade Federal de Sergipe, sob o parecer 1.849.207.

\section{RESULTADOS}

Participaram do estudo 75 caminhoneiros, do sexo masculino, com idade entre 19 e 60 anos, 43 $(57,3 \%)$ eram casados, católicos 54 (72\%), com renda mensal de mais de 2 salários mínimos 50 (66,6\%), residentes em Sergipe 39 (52\%) e com mais de 10 anos de profissão 49 (65,3\%). A escolaridade de 32 $(42,6 \%)$ caminhoneiros estava entre ensino médio incompleto e completo (Tabela 1$)$.

Tabela 1 - Perfil sociodemográfico dos caminhoneiros. Itabaiana, SE, Brasil, 2017 (continua)

\begin{tabular}{|c|c|c|c|}
\hline Características & & $(n=75)$ & $(\%)$ \\
\hline \multirow[t]{5}{*}{ Idade } & De 19 a 29 anos & 10 & 13,3 \\
\hline & De 30 a 39 anos & 31 & 41,3 \\
\hline & De 40 a 49 anos & 23 & 30,6 \\
\hline & De 50 a 59 anos & 10 & 13,3 \\
\hline & 60 ou mais & 1 & 1,3 \\
\hline \multirow[t]{3}{*}{ Tempo de profissão } & Mais de 10 anos & 49 & 65,3 \\
\hline & Menos de 10 anos & 15 & 20 \\
\hline & Igual 10 anos & 11 & 14,6 \\
\hline \multirow[t]{6}{*}{ Escolaridade } & $1^{\mathrm{a}}$ a $4^{\mathrm{a}}$ do EF & 7 & 9,3 \\
\hline & $5^{\mathrm{a}}$ a $8^{\mathrm{a}}$ do $\mathrm{EF}$ & 28 & 37,3 \\
\hline & EF completo & 4 & 5,3 \\
\hline & Ensino médio incompleto & 11 & 14,6 \\
\hline & Ensino médio completo & 21 & 28 \\
\hline & Superior incompleto & 4 & 5,3 \\
\hline \multirow[t]{4}{*}{ Renda mensal } & 1 salário mínimo & 5 & 6,6 \\
\hline & 1 a 2 salários & 20 & 26,6 \\
\hline & 2 a 3 salários & 23 & 30,6 \\
\hline & 3 ou mais & 27 & 36 \\
\hline
\end{tabular}




\begin{tabular}{|c|c|c|c|}
\hline \multirow[t]{3}{*}{ Religião } & Católico & 54 & 72 \\
\hline & Protestante/Evangélico & 9 & 12 \\
\hline & Nenhuma & 12 & 16 \\
\hline \multirow[t]{4}{*}{ Situação conjugal } & Solteiro & 12 & 16 \\
\hline & Parceiro fixo & 14 & 18,6 \\
\hline & Divorciado & 6 & 8 \\
\hline & Casado & 43 & 57,3 \\
\hline \multirow[t]{3}{*}{ Local de Residência } & Sergipe & 39 & 52 \\
\hline & Bahia & 11 & 14,6 \\
\hline & Outros Estados & 25 & 33,3 \\
\hline \multirow[t]{3}{*}{ Trajetos rotineiros } & Bahia & 27 & 36 \\
\hline & São Paulo & 22 & 29,3 \\
\hline & Outros* & 26 & 34,6 \\
\hline \multirow[t]{4}{*}{ Tempo médio fora da residência } & Até 9 dias & 25 & 33,3 \\
\hline & De 10 a 19 dias & 22 & 29,3 \\
\hline & De 20 a 29 dias & 12 & 16 \\
\hline & 30 dias ou mais & 16 & 21,3 \\
\hline
\end{tabular}

*Alagoas, Pernambuco, Minas Gerais, Rio de Janeiro, Pará e Paraná.

Sobre o conhecimento das IST, $74(98,6 \%)$ demonstraram adequado saber. No que se refere às IST que conheciam, todos citaram a Aids e 35 (46,6\%) a hepatite. Quanto aos veículos de informação, os mais citados foram televisão, rádio e campanhas na estrada.

Sobre o uso de preservativo, 72 (96\%) concordaram que era a melhor maneira de evitar a transmissão das IST, 45 (60\%) não sabiam dizer cuidados necessários para o uso correto e 72 (96\%) conheciam o preservativo feminino (Tabela 2).

Tabela 2 - Conhecimento de caminhoneiros acerca das Infecções Sexualmente Transmissíveis. Itabaiana, SE, Brasil, 2017 (continua)

\begin{tabular}{|c|c|c|c|}
\hline & & (n) & $(\%)$ \\
\hline \multirow[t]{2}{*}{ Sabe o que é IST e como é transmitida? } & Sim & 74 & 98,6 \\
\hline & Não & 1 & 1,4 \\
\hline \multirow{4}{*}{$\begin{array}{l}\text { Qual(s) IST uma pessoa pode ser infectada se não usar } \\
\text { preservativos em relações sexuais? }\end{array}$} & Aids & 75 & 100 \\
\hline & Sífilis & 71 & 94,6 \\
\hline & Gonorreia & 67 & 89,3 \\
\hline & Hepatite & 35 & 46,6 \\
\hline \multirow{5}{*}{$\begin{array}{l}\text { Veículo de informação que normalmente recebe informação } \\
\text { sobre as IST? }\end{array}$} & TV & 60 & 80 \\
\hline & Campanhas nas Estradas & 60 & 80 \\
\hline & Rádio & 45 & 60 \\
\hline & Jornais e revistas & 28 & 37,4 \\
\hline & Postos de Saúde & 23 & 30,6 \\
\hline \multicolumn{4}{|l|}{ Concordaram com a afirmação: } \\
\hline \multirow{2}{*}{$\begin{array}{l}\text { "O risco de transmissão do HIV/Aids, pode ser reduzido, } \\
\text { se uma pessoa tiver relações sexuais somente com parceiro } \\
\text { fixo e não infectado". }\end{array}$} & Sim & 71 & 94,6 \\
\hline & Não & 4 & 5,4 \\
\hline \multirow{2}{*}{$\begin{array}{l}\text { Concordaram com a afirmação: "Uma pessoa com aparência } \\
\text { saudável pode estar com uma IST, como a Aids." }\end{array}$} & Sim & 64 & 85,4 \\
\hline & Não & 11 & 14,6 \\
\hline
\end{tabular}




\begin{tabular}{llccc}
\hline Conhece algum serviço de saúde em que o teste para Aids & Sim & 51 & 68 \\
\cline { 2 - 4 } é feito gratuitamente? & Não & 24 & 32 \\
\hline $\begin{array}{l}\text { Concordaram com a afirmação: "Usar preservativo é } \\
\text { melhor maneira de evitar a transmissão de IST". }\end{array}$ & Sim & 72 & 96 \\
\cline { 2 - 4 } & Não & 3 & 4 \\
\hline Conhece o preservativo feminino? & Sim & 72 & 96 \\
\cline { 2 - 4 } & Não & 30 & 4 \\
\hline $\begin{array}{l}\text { Sabe dizer cuidados necessários para o uso correto do do } \\
\text { preservativo? }\end{array}$ & Sim & 45 & 60 \\
\cline { 2 - 4 } & Não &
\end{tabular}

Na observação das atitudes e práticas, verifica-se que 18 (24\%) tiveram ou têm alguma IST, 29 (38,6\%) acham que têm comportamento de risco para contrair IST, 65 (86,6\%) compraram preservativo, 36 (48\%) possuíam preservativo no caminhão ou na casa no momento da entrevista. Apenas $17(22,6 \%)$ utilizaram preservativo na última relação sexual e dos que possuíam parceiros casuais $28(57,14 \%)$ relataram usar preservativo nas relações sexuais com esses parceiros (Tabela 3).

Tabela 3 - Atitudes e práticas de caminhoneiros acerca das Infecções Sexualmente Transmissíveis. Itabaiana, SE, Brasil, 2017 (continua)

\begin{tabular}{|c|c|c|c|}
\hline & & (n) & $(\%)$ \\
\hline \multirow[t]{6}{*}{ Já teve ou tem alguma IST? } & Sim & 18 & 24 \\
\hline & Não & 57 & 76 \\
\hline & Gonorreia & 14 & 77,7 \\
\hline & Herpes & 2 & 11,1 \\
\hline & Sífilis & 1 & 5,55 \\
\hline & Cancro Mole & 1 & 5,55 \\
\hline \multirow{2}{*}{$\begin{array}{l}\text { Acha que tem comportamento de risco para contrair } \\
\text { IST? }\end{array}$} & Sim & 29 & 38,66 \\
\hline & Não & 46 & 61,34 \\
\hline \multirow[t]{2}{*}{ Já fez o teste para diagnóstico do HIV/Aids? } & Sim & 45 & 60 \\
\hline & Não & 30 & 40 \\
\hline \multirow[t]{2}{*}{ Já comprou camisinha? } & Sim & 65 & 86,6 \\
\hline & Não & 10 & 13,4 \\
\hline \multirow[t]{2}{*}{ Já recebeu/pegou camisinha de graça? } & Sim & 72 & 96 \\
\hline & Não & 3 & 4 \\
\hline \multirow{2}{*}{$\begin{array}{l}\text { No momento, tem camisinha no seu caminhão ou na } \\
\text { sua casa? }\end{array}$} & Sim & 36 & 48 \\
\hline & Não & 39 & 52 \\
\hline \multirow[t]{2}{*}{ Na última relação sexual usou camisinha? } & Sim & 17 & 22,6 \\
\hline & Não & 58 & 77,4 \\
\hline \multirow{3}{*}{$\begin{array}{l}\text { Usa camisinha nas relações sexuais com a }(0) \text { parceira(o) } \\
\text { fixa(o)? }\end{array}$} & Sim & 3 & 5,26 \\
\hline & Não & 51 & 89,48 \\
\hline & Esporadicamente & 3 & 5,26 \\
\hline \multirow[t]{3}{*}{ Porque não utiliza camisinha? } & Confia no parceiro(a) & 52 & 69,33 \\
\hline & Diminuir ereção & 13 & 17,33 \\
\hline & Diminuir sensibilidade e prazer & 10 & 13,34 \\
\hline \multirow{2}{*}{$\begin{array}{l}\text { Teve relação sexual com parceiras(os) casuais nos } \\
\text { últimos } 12 \text { meses? }\end{array}$} & Sim & 49 & 65,34 \\
\hline & Não & 26 & 34,66 \\
\hline \multirow{2}{*}{$\begin{array}{l}\text { Teve mais que cinco parceiros(a) sexuais nos últimos } \\
12 \text { meses? }\end{array}$} & Sim & 17 & 22,6 \\
\hline & Não & 58 & 77,4 \\
\hline
\end{tabular}




\begin{tabular}{llll}
\hline \multirow{2}{*}{$\begin{array}{l}\text { Usa camisinha nas relações sexuais com parceiras(os) } \\
\text { casuais? }\end{array}$} & Sim & 28 & 57,16 \\
\cline { 2 - 3 } & Não & 12 & 24,48 \\
\cline { 2 - 3 } & Esporadicamente & 9 & 18,36 \\
\hline \multirow{2}{*}{$\begin{array}{l}\text { Já teve relações sexuais com uma mulher que estava } \\
\text { usando o preservativo feminino? }\end{array}$} & Sim & 11 & 14,6 \\
\cline { 2 - 3 } & Não & 64 & 85,4
\end{tabular}

\section{- DISCUSSÃO}

Conforme os resultados, a totalidade de entrevistados foi do sexo masculino reproduzindo sua característica histórica, exercida quase exclusivamente por homens. Estudos em Vitória da Conquista$\mathrm{BA}^{(10)}$ e em Goiânia-GO ${ }^{(14)}$ mostram que a maioria dos caminhoneiros era casado ou tinha parceira fixa e que grande parte relatou que no último ano tiveram relações sexuais com parceiros casuais, principalmente enquanto estavam na estrada, durante suas viagens.

Os caminhoneiros relataram fazer viagens para outros estados e percorrerem rotas consideradas longas, com mais de $50 \mathrm{~km}$, passando em média 19,6 dias fora de suas residências, o que pode contribuir para exposição e aquisição de IST, assim como na disseminação destas ${ }^{(9)}$.

No tocante ao conhecimento acerca das IST e suas formas de transmissão, foi possível identificar que a quase totalidade dos caminhoneiros entrevistados possuíam conhecimento sobre o assunto, fato que pode ser justificado pela facilidade de acesso às informações, possibilitando melhor conhecimento a respeito do tema. Sobre quais às IST que conheciam, todos citaram a Aids e apenas 35 $(46,6 \%)$ a hepatite. Inquérito realizado com 64 indivíduos jovens e adultos em Recife-PE e São PauloSP identificou que a hepatite assim como outras IST ainda são pouco conhecidas por esse público ${ }^{(15)}$.

As informações sobre IST chegam principalmente por meio de meios de comunicação de massa, como televisão e rádio, que são os mais acessíveis à população, como corroborado em pesquisas nacionais ${ }^{(9,15)}$. Foi identificado também que a maioria dos caminhoneiros recebeu informações durante campanhas nas estradas, o que demonstra que com o passar dos anos as políticas públicas de prevenção às IST estão sendo estimuladas.

Nesse contexto, homens podem vivenciar essa realidade de modo diferente das mulheres, uma vez que apresentam maior resistência em relação à busca de orientações adequadas sobre prevenção e não se consideram vulneráveis.

Foi observado que, apesar dos caminhoneiros identificarem que o preservativo é a melhor maneira de evitar a transmissão das IST, quase $25 \%$ não utilizava em sua prática, sugerindo não haver uma associação entre o conhecimento e a prática sexual.

Como justificativa para o não uso do preservativo durante as relações sexuais com parceiros fixos, muitos relataram a confiança na parceria, a diminuição da ereção, da sensibilidade e do prazer quando estavam utilizando preservativo, justificativa que também foi encontrada no estudo realizado com caminhoneiros na Bahia ${ }^{(10)}$.

Torna-se evidente a baixa adesão em relação ao uso do preservativo, comportamento que pode estar relacionado a crenças como a de que o preservativo prejudica o desempenho sexual e a de que o sexo com parceiro(a) aparentemente saudável não envolve risco de contaminação. Ademais, estudo realizado em Santos-SP identificou que a utilização do preservativo se apresenta de forma diferenciada segundo o parceiro sexual ${ }^{(9)}$.

Portanto, o parceiro fixo pode estar vulnerável no que se refere às IST, visto que foi identificado que durante as relações sexuais com parceiro fixo a taxa de adesão ao preservativo é pequena, e caminhoneiros relataram não usar preservativo nas relações com parceiros casuais.

Foi possível verificar que a maioria dos caminhoneiros, em algum momento da vida, já tinha comprado ou recebido preservativo gratuitamente, porém no momento da entrevista grande parte 
não possuía o preservativo no caminhão, em casa ou consigo. Relataram, ainda, não ter conhecimento acerca dos cuidados necessários para o uso correto do preservativo, fato que chama atenção, pois o uso incorreto do preservativo pode acarretar no aumento do risco para a aquisição das IST ${ }^{(8)}$.

No tocante ao conhecimento sobre o preservativo feminino, a maioria relatou conhecer, porém não utilizam em sua prática sexual. Este fato pode ser justificado pelo alto custo do preservativo feminino quando comparado ao masculino e a distribuição gratuita por toda a rede pública ser menor. Em tempo, fatores como as relações de poder entre homens e mulheres interferem diretamente no uso ou não do preservativo, e influenciam sobremaneira na adesão ao método pelo casal ${ }^{(15)}$.

As atitudes e práticas dos caminhoneiros pesquisados evidenciou comportamento de risco para contrair algum tipo de IST, pois $18(24 \%)$ relataram já ter adquirido alguma IST na vida, contrapondo à taxa brasileira de homens sexualmente ativos entre 15 e 64 anos que declararam ter tido pelo menos um antecedente relacionado às IST com aproximadamente $17 \%{ }^{(6)}$.

O presente estudo apresentou limitações por ter sido desenvolvido com caminhoneiros residentes apenas do estado de Sergipe, fato que expõe a representação de uma população específica com características sociais e culturais próprias. Ademais, não se verificou conhecimentos, atitudes e práticas de suas parceiras fixas e/ou casuais a fim de estabelecer discussão sobre relações do casal com o uso do preservativo.

\section{CONCLUSÃO}

A maioria dos caminhoneiros possui conhecimento adequado acerca das IST, HIV/Aids, especialmente sobre suas formas de transmissão e prevenção. Esse conhecimento foi adquirido por diferentes fontes de informação, porém não esteve proporcionalmente alinhado com as atitudes e as práticas do grupo estudado, haja vista um número considerável de caminhoneiros que já adquiriram IST e a baixa adesão ao uso do preservativo como prática sexual.

Existe a necessidade de melhores estratégias de informação e comunicação para essa população com foco nas formas de prevenção das IST e na importância do uso do preservativo. É desejável que as lacunas nas atitudes e práticas observadas nesse estudo possam contribuir na identificação de pontos a serem desenvolvidos e enfatizados no planejamento em saúde e em estratégias de intervenção para promover saúde desse grupo populacional que vivencia contexto de maior vulnerabilidade.

Sugere-se que outros estudos sejam realizados com caminhoneiros que residem em outras regiões do Brasil, a fim de se comparar conhecimentos, atitudes e práticas sobre do uso do preservativo em diferentes contextos, bem como com as parceiras sexuais destes profissionais. Estudos nessa perspectiva poderão desvelar questões culturais e de gênero relacionadas à baixa adesão ao uso do preservativo entre caminhoneiros.

\section{REFERÊNCIAS}

1. Ministério da Saúde (BR). Secretaria de Vigilância em Saúde. Departamento de DST, Aids e Hepatites Virais. Protocolo Clínico e Diretrizes Terapêuticas para Atenção Integral às Pessoas com Infecções Sexualmente Transmissíveis. Brasília: Ministério da Saúde; 2015.

2. Rocha EM. DST e AIDS em regiões de fronteiras: um estudo com caminhoneiros no estado de Rondônia [dissertação]. Brasília (DF): Universidade de Brasília; 2008.

3. Escola Nacional de Saúde Pública Sergio Arouca (Ensp). Saúde em Números: 30 milhões de mortes. Revista RADIS. [Internet] 2016;(167) [acesso em 26 set 2016]. Disponível: http://www6.ensp.fiocruz.br/radis/sites/default/ files/radis_167_web.pdf.

4. Ministério da Saúde (BR). Secretaria de Vigilância em Saúde. Departamento de DST, Aids e Hepatites Virais. Boletim Epidemiológico - Aids e DST. Ano V - nº 01. Brasília: Ministério da Saúde; 2016. 
5. Ministério da Saúde (BR). Secretaria de Vigilância em Saúde. Departamento de DST, Aids e Hepatites Virais. Boletim Epidemiológico - Aids e DST. Ano IV - nº 01. Brasília: Ministério da Saúde; 2015.

6. Ministério da Saúde (BR). Secretaria de Vigilância em Saúde. Departamento de DST, Aids e Hepatites Virais. Pesquisa de conhecimento, atitudes e práticas na população brasileira. Brasília: Ministério da Saúde; 2011.

7. Aragão JCS, Lopes CS, Bastos Fl. Comportamento Sexual de estudantes de um Curso de Medicina do Rio de Janeiro. Rev. bras. educ. med. [Internet] 2011;35(3) [acesso em 17 jul 2016]. Disponível: http://dx.doi.org/10.1590/ S0100-55022011000300006.

8. de Sousa LB, Cunha DFF, Ximenes LB, Pinheiro AKB, Vieira NFC. Conhecimentos, atitudes e prática de mulheres acerca do uso do preservativo. Rev. enferm. UERJ. [Internet] 2011;19(1) [acesso em 17 jul 2016]. Disponível: http:// www.facenf.uerj.br/v19n1/v19n1a24.pdf.

9. Villarinho L, Bezerra I, Lacerda R, Latorre MRDO, Paiva V, Stall R, et al. Caminhoneiros de rota curta e suavulnerabilidade ao HIV, Santos, SP. Rev. Saúde Pública. [Internet] 2002;36(4) [acesso em 17 jul 2016]. Disponível: http://dx.doi.org/10.1590/S0034-89102002000500009.

10. de Sousa LMS, Silva LS, Palmeira AT. Representações sociais de caminhoneiros de rota curta sobre HIV/AIDS. Psicol. Soc. [Internet] 2014;26(2) [acesso em 17 jul 2016]. Disponível: http://www.ufrgs.br/seerpsicsoc/ojs2/index. php/seerpsicsoc/article/view/3755.

11. Brasil. Lei n. 13.044, de 19 de novembro de 2014. Confere ao Município de Itabaiana no Estado de Sergipe o título de Capital Nacional do Caminhão. [Internet] Brasília, DF, Diário Oficial da União, 2014 [acesso em 07 jul 2016]. Disponível: http://www.planalto.gov.br/ccivil_03/_Ato2011-2014/2014/Lei/L13044.htm.

12. Brasil. Projeto de Lei 7132/2014 do Senado. Ementa: Confere ao Município de Itabaiana, no Estado de Sergipe, o título de Capital Nacional do Caminhão. [Internet] Brasília, DF, 2014 [acesso em 07 jul 2016]. Disponível: http:// www.camara.gov.br/proposicoesWeb/fichadetramitacao?idProposicao=606303.

13. Ministério da Saúde (BR). Conselho Nacional de Saúde. Diretrizes e normas regulamentadoras de pesquisa envolvendo seres humanos. Resolução n. 466, de 12 de dezembro de 2012. Brasília; 2012.

14. Teles AS, de Matos MA, Caetano KAA, Costa LA, França DDS, Pessoni GC, et al. Comportamentos de risco para doenças sexualmente transmissíveis em caminhoneiros no Brasil. Rev Panam Salud Publica. [Internet] 2008;24(1) [acesso em 07 jul 2016]. Disponível: http://www.scielosp.org/pdf/rpsp/v24n1/v24n1a03.pdf.

15. Garcia S, de Souza FM. Vulnerabilidades ao HIV/AIDS no contexto brasileiro: iniquidades de gênero, raça e geração. SP, Saúde Soc. [Internet] 2010;19(2) [acesso em 07 jul 2016]. Disponível: http://dx.doi.org/10.1590/S010412902010000600003. 